\title{
Oxygen-Guided Radiation Therapy
}

\author{
Boris Epel, PhD ${ }^{*}$, , Matthew C. Maggio, BS ${ }^{*}, \dagger$, Eugene D. Barth, BA ${ }^{*}, \dagger$, Richard C. Miller, \\ $\mathrm{PhD}^{*}, \dagger$, Charles A. Pelizzari, $\mathrm{PhD}^{\star}, \dagger$, Martyna Krzykawska-Serda, PhD ${ }^{\star}, \dagger$, Subramanian V. \\ Sundramoorthy, $\mathbf{M S}^{*}, \dagger$, Bulent Aydogan, $\mathrm{PhD}^{\dagger}$, Ralph R. Weichselbaum, $\mathbf{M D}^{\star}, \dagger, \ddagger$, Victor $\mathbf{M}$. \\ Tormyshev, $\mathrm{PhD}^{\star}, \S, \|$, and Howard J. Halpern, MD, PhD ${ }^{*}$, \\ * National Institutes of Health Center for EPR Imaging In Vivo Physiology, University of Chicago, \\ Chicago, Illinois \\ †Department of Radiation and Cellular Oncology, University of Chicago, Chicago, Illinois \\ ‡Ludwig Center for Metastasis Research, University of Chicago, Chicago, Illinois \\ $\S$ Novosibirsk Institute of Organic Chemistry, Novosibirsk, Russia \\ "Novosibirsk State University, Novosibirsk, Russia
}

\section{Summary}

This article presents the first experimental demonstration that electron paramagnetic resonance oxygen image guided radiation therapy increases tumor control with hypoxic boost doses relative to well-oxygenated tumor boosts of roughly the same volume in mouse fibrosarcoma models. This is the first demonstration of the effectiveness of targeting specific hypoxic tumor in mammalian systems.

Purpose: It has been known for over 100 years that tumor hypoxia, a near-universal characteristic of solid tumors, decreases the curative effectiveness of radiation therapy. However, to date, there are no reports that demonstrate an improvement in radiation effectiveness in a mammalian tumor on the basis of tumor hypoxia localization and local hypoxia treatment.

Methods and Materials: For radiation targeting of hypoxic subregions in mouse fibrosarcoma, we used oxygen images obtained using pulse electron paramagnetic resonance $\mathrm{pO}_{2}$ imaging combined with 3D-printed radiation blocks. This achieved conformal radiation delivery to all hypoxic areas in FSa fibrosarcomas in mice.

Results: We demonstrate that treatment delivering a radiation boost to hypoxic volumes has a significant $(P=.04)$ doubling of tumor control relative to boosts to well-oxygenated volumes. Additional dose to well-oxygenated tumor regions minimally increases tumor control beyond the $15 \%$ control dose to the entire tumor. If we can identify portions of the tumor that are more resistant to radiation, it might be possible to reduce the dose to more sensitive tumor volumes without significant compromise in tumor control.

Reprint requests to: Howard J. Halpern, MD, PhD, Department of Radiation and Cellular Oncology, University of Chicago, MC1105, 5841 S. Maryland Ave., Chicago, IL 60637. Tel: (773) 702-6871; h-halpern@ uchicago.edu.

Conflict of interest: U.S. patent 8,664,955 has been awarded to H.H. and B.E. for $\mathrm{pO}_{2}$ imaging methodology; they are also owners of a start-up company, O2M Technologies, LLC, (Chicago, IL) that markets the $\mathrm{pO}_{2}$ imaging technology.

Supplementary material for this article can be found at https://dx.doi.org/10.1016/j.ijrobp.2018.10.041. 
Conclusions: This work demonstrates in a single, intact mammalian tumor type that tumor hypoxia is a local tumor phenomenon whose treatment can be enhanced by local radiation. Despite enormous clinical effort to overcome hypoxic radiation resistance, to our knowledge this is the first such demonstration, even in preclinical models, of targeting additional radiation to hypoxic tumor to improve the therapeutic ratio.

\section{Introduction}

Radiation therapy is delivered to approximately $60 \%$ of patients with cancer, but it often does not achieve local control. ${ }^{1}$ Technically advanced intensity modulated radiation therapy (IMRT) can deliver radiation with sharp spatial dose gradients over fractions of a centimeter in human tumors, allowing more conformal and increased whole tumor dose while sparing nearby critical normal organs and tissues. ${ }^{2,3}$ IMRT might also enable customized treatments that take into account differential sensitivity of portions of tumors to radiation. However, currently, there is no established noninvasive clinical imaging method that locates radiation resistant tumor portions. ${ }^{4,5}$ Although the technique of the current study does not involve IMRT, the identification of all radiation-resistant tumor subvolumes, if translated to the clinic, will enable dose painting to enhance IMRT effectiveness.

The current success of IMRT is the result of its fulfillment of the first of its 2 promises: its capacity to spare critical structures. The present inability to define variably sensitive tumor subregions has led to the specification of limits on inhomogeneity of the planning target volume (PTV) for clinicians. ${ }^{4}$ This is a statement of our ignorance in our definition of variable sensitivity within our treatment volumes. The identification of these radiationresistant tumor portions is crucial to this second promise: intratumoral dose painting to focus dose on resistant tumor subvolumes with the possibility to reduce the dose to the more sensitive portions of tumor while maintaining overall control.

Hypoxia is a characteristic feature of most solid tumors, ${ }^{6}$ and is a result of chaotic tumor vasculature stimulated by chaotic tumor growth ${ }^{7,8}$ and tumor oxygen demand. ${ }^{9}$ Poor radiation therapy outcomes have been observed in patients with high hypoxic fractions in cancers of the head and neck, ${ }^{10}$ soft tissue sarcoma, ${ }^{11}$ and uterine cervix. ${ }^{12}$ Hypoxic sensitizers ultimately failed to provide clinical benefits because of the lack of effectiveness and toxicity, although the assumption that all tumors were hypoxic and the lack of attention to exclusion of well-oxygenated tumors from studies diluted the potential benefit from human sensitizer trials. ${ }^{13}$ Meta-analysis of head and neck hypoxia modification shows benefit. ${ }^{14}$ Radiotracers such as ${ }^{18} \mathrm{~F}$-misonidazole have been developed that are selectively metabolized and entrapped within hypoxic tissue, allowing spatial identification of subregions of tumor hypoxia identified using positron emission tomography (PET) images. ${ }^{15,16}$ Correlation of early ${ }^{18} \mathrm{~F}$-EF5-PET response in preclinical models with improved longterm tumor control with hypoxia toxin plus radiation versus either treatment alone ${ }^{17}$ provides indication of PET's ability to predict whole tumor response, validating the TransTasmin clinical trial. ${ }^{18}$ Recent data show repeatability of hypoxia PET images. ${ }^{19}$ However, no data showing improved tumor control from targeting hypoxic regions defined by PET images have been published in humans or preclinical models. A recent human phase 2 trial in non-small cell lung cancer using ${ }^{18} \mathrm{~F}$-misonidazole PET failed to demonstrate any benefit 
from a hypoxic boost of $20 \mathrm{~Gy}$ of radiation added to a tumor dose of $66 \mathrm{~Gy} .{ }^{20}$ This result is possibly because of inaccuracy in the PET quantification used to identify and delineate the relevant hypoxia targets in lung - a site that requires sophisticated respiratory gating techniques - as a result of respiratory lesion motion. ${ }^{21}$ Might hypoxia and its resistance to radiation be a general characteristic of all cancer cells, resistant to local therapy and a fortiori extra radiation directed to a part of the tumor, because, for example, all parts of the tumor are transiently hypoxic? ${ }^{22}$

Fluorine-18 labeled PET radiotracers, such as FMISO, EF5, and HX4, have the potential to visualize hypoxia distributions within tumor, but they possess a number of disadvantages: (1) These tracers do not provide high-contrast images, making them difficult to interpret; (2) the studies take much longer (2-4 hours) than conventional fluorodeoxyglucose scans because of the slow washout of the radiotracer from nonhypoxic tissue; (3) radiotracer techniques possess limited temporal resolution and are suboptimal for measuring acute changes in the hypoxia status of tumors; and (4) in human subjects, PET-defined hypoxia has sufficiently limited spatial resolution and thus hypoxic and well-oxygenated tumor are averaged. ${ }^{23}$

In this work, we propose an alternative imaging modality with the potential to provide a more timely and accurate quantitative way to determine subregional tumor hypoxia. This is demonstrated in the hypoxic subregions of tiny ( $400 \mu \mathrm{L}$ mean magnetic resonance imaging [MRI] volume) leg-borne FSa fibrosarcomas in mice using electron paramagnetic resonance

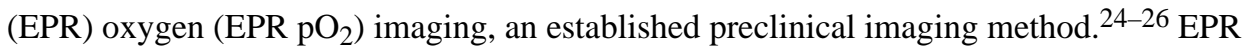
imaging is conceptually similar to nuclear MRI, but it detects signals from unpaired electron spins of injected stable, nontoxic, paramagnetic spin probe reporters. The electron spinlattice relaxation rate of these reporters is a linear function of local $\mathrm{pO}_{2}$. Noninvasiveness, high accuracy (1 torr at low $\mathrm{pO}_{2}$ ), good spatial resolution $(\sim 1 \mathrm{~mm})$, and a lack of confounding variability ${ }^{27}$ make EPR imaging a valuable tool for preclinical studies. For animals with legborne FSa fibrosarcomas treated to a variety of radiation doses, tumor control correlated significantly not only with dose but with EPR-defined hypoxic fraction. ${ }^{28}$ EPR $\mathrm{pO}_{2}$-based hypoxic fraction correlates with the concentration of the hypoxia protein vascular endothelial growth factor. ${ }^{29} \mathrm{EPR} \mathrm{pO}_{2}$-based fraction of tumor volume with $\mathrm{pO}_{2}$ less than 10 torr (HF10) predicts tumor control in FSa fibrosarcoma and MCa4 mammary carcinomas treated to a $50 \%$ total control dose $\left(\mathrm{TCD}_{50}\right)$. With $\mathrm{HF} 10$ less than $10 \%$ or $15 \%$, $90 \%$ of tumors were controlled, whereas tumors with higher HF10 treated to the $\mathrm{TCD}_{50}$ were controlled in only $37 \%$ or $23 \%$ of tumors, respectively. ${ }^{30}$ The extent of antiangiogenic sunitinib enhancement of radiation growth delay correlates with EPR $\mathrm{pO}_{2}$ oxygenation. ${ }^{31}$

Using the combination of $\mathrm{pO}_{2}$ EPR imaging, conformal radiation enabled by 3D-printed radiation blocks, and a clonogenic 90-day tumor control endpoint, we provide to our knowledge the first evidence that selective hypoxia targeting significantly enhances local tumor control.

\section{Experimental strategy}

We hypothesize that there is an advantage to treating subregions of a tumor that were defined as hypoxic ( $\mathrm{pO}_{2} \leq 10$ torr) by $\mathrm{EPR} \mathrm{pO}_{2}$ images and thus as resistant to radiation. We 
first treated the whole tumor to a dose that was determined in separate experiments to control 15\% of tumors (a TCD 15 ; Fig. E1, available online at https://dx.doi.org/10.1016/ j.ijrobp.2018.10.041 ). For FSa mouse fibrosarcomas the $\mathrm{TCD}_{15}$ and the $\mathrm{TCD}_{95}$ were determined to be 22.5 and $35.5 \mathrm{~Gy}$, respectively. We tested our hypothesis by the administration of additional radiation, a boost of $13 \mathrm{~Gy}\left(\mathrm{TCD}_{95}-\mathrm{TCD}_{15}\right)$ to all hypoxic subregions of a tumor that are hypothesized to be resistant. This was compared in a randomized study with treatment of well-oxygenated regions with a 13-Gy boost of roughly equal volume. It was intended that all tumors would be treated to the same integral dose. Thus, the only difference between tumor treatments was the boost location based on oxygenation distribution determined by the $\mathrm{EPR} \mathrm{pO}_{2}$ image.

\section{Methods and Materials}

\section{Experimental plan}

In addition to the usual radiation therapy volumes of gross tumor volume (GTV), clinical target volume, GTV plus the additional volume at high risk for subclinical cancer, and the PTV (the clinical target volume plus a margin for tumor location uncertainties and subject setup uncertainties), we define high-risk radiation target volumes. Following Lin et al, ${ }^{32}$ the hypothesized resistant hypoxic high-risk subvolumes will be designated the $\mathrm{GTV}_{\mathrm{HR}}$. The additional volume around and including the $\mathrm{GTV}_{\mathrm{HR}}$ to account for image resolution and other subject setup uncertainties will be referred to as the planning high-risk volume $\left(\mathrm{PTV}_{\mathrm{HR}}\right)$. The hypothesized low-risk, well-oxygenated subvolumes will be designated the $G V_{L R}$. The additional volume around and including the $G_{T V} V_{L R}$ to account for image resolution and other subject setup uncertainties will be referred to as the planning low-risk volume $\left(\mathrm{PTV}_{\mathrm{LR}}\right)$.

Animals were treated with a whole tumor (PTV) radiation dose of $22.5 \mathrm{~Gy}$, which controlled, in separate experiments, $15 \%$ of tumors of the same size (Fig. E1; available online at https://dx.doi.org/10.1016/j.ijrobp.2018.10.041). Animals were then randomized to receive 13-Gy boosts delivered to either all $\mathrm{EPR} \mathrm{pO}_{2}$ image-based hypoxic voxels $\left(\mathrm{pO}_{2} \leq 10\right.$ torr), the $\mathrm{PTV}_{\mathrm{HR}}$, or an equal volume that was well oxygenated ( $\mathrm{pO}_{2}>10$ torr), the $\mathrm{PTV}_{\mathrm{LR}}$. Fiftyfour treated animals were randomized into the 2 experimental groups and followed until they survived the 90-day period of observation after treatment, failed because of tumor regrowth, or were censored for metastasis or other unrelated causes. Tumor regrowth was scored at the time at which the tumor exceeded twice the initial volume measured with calipers. Animals were then immediately euthanized.

\section{Animal model and anesthesia}

The animals used were $\mathrm{C} 3 \mathrm{H} / \mathrm{HeNCrl}$ mice. For imaging, anesthesia was induced using $2 \%$ isoflurane mixed with $21.5 \%$ oxygen/78.5\% nitrogen (air) and maintained with $1.5 \%$ isoflurane and air, administered via mask. Anesthesia depth was guided by respiration rate, 1.5 to $2 \mathrm{~Hz}$. The spin probe was delivered intravenously through the tail vein and collected by intravesical cannulation. ${ }^{33}$ Euthanasia was performed with isoflurane overdose or $\mathrm{CO}_{2}$ asphyxiation, with confirmation by cervical dislocation. Animal experiments followed U.S. Public Health Service policy, National Institutes for Health Guide for the Care and Use of 
Laboratory Animals and were approved by the Institutional Animal Care and Use Committee.

\section{Tumor model}

Syngeneic FSa fibrosarcoma tumor cells ${ }^{34}$ were grown in mouse flanks to $2 \mathrm{~cm}$ in diameter from sixth-generation frozen cells obtained from Kathryn Mason at M.D. Anderson Hospital. The cells were frozen and then regrown in mice to $1 \mathrm{~cm}$ diameter, where they were harvested. A suspension of 0.1 to $4.0 \times 10^{6}$ cells in modified Eagle medium with $10 \%$ fetal bovine serum were grown in the gastrocnemius muscle of the legs of 10-week-old female $\mathrm{C} 3 \mathrm{H}$ mice to a median volume of $400 \mu \mathrm{L}$, as defined on MRI.

\section{Imaging}

For all animals, 3 images were obtained before treatment: an MRI to define the 3dimensional (3D) tumor boundary in the mouse leg, an $\mathrm{EPR} \mathrm{pO}_{2}$ image to define the location of hypoxic voxels within the tumor, and a computed tomography (CT) image in the XRAD225Cx with the same $x$-ray tube to be used for treatment. These images were registered with each other so that the information from all of them could be incorporated in a treatment plan to deliver 3D conformal radiation to the hypoxic or well-oxygenated tumor regions. T2-weighted MRI was acquired with a 9.4-T small animal scanner (Bruker, Billerica, MA) using a multislice RARE (Rapid Imaging with Refocused Echoes sequence. ${ }^{35} \mathrm{EPR}$ partial oxygen pressure $\mathrm{pO}_{2}$ images were acquired with spin-lattice relaxation oxygen imaging ${ }^{27}$ in 10 minutes using a $250-\mathrm{MHz}$ pulse EPR imager. ${ }^{36,37} \mathrm{pO}_{2}$ was imaged with $135 \mu \mathrm{L}$ of an $80-\mathrm{mM}$ solution of the OX63- $\mathrm{d}_{24}$ oxygen-measuring spin probe $(0.43$ $\mathrm{mmol} / \mathrm{kg}$ ) as an intravenously injected bolus followed by $3.5 \mu \mathrm{L} / \mathrm{min}$ continuous infusion over 30 minutes during $3 \mathrm{pO}_{2}$ images $(0.45 \mathrm{mmol} / \mathrm{kg}$ or a total of $0.9 \mathrm{mmol} / \mathrm{kg})$. OX63- $\mathrm{d}_{24}$ is a partially deuterated trisodium salt of methyl-tris [8-carboxy-2,2,6,6-tetrakis[2hydroxy- $1^{2} \mathrm{H}$-ethyl]benzo [1,2-d:4,5-d']bis [1,3]dithiol-4-yl]- trisodium salt, MW 1451, synthesized by the Novosibirsk Institute of Organic Chemistry, ${ }^{38}$ also available as OX71 from GE Health Care (Little Chalfont, Buckinghamshire, United Kingdom). The $\mathrm{LD}_{50}$ for mice is $8 \mathrm{mmol} / \mathrm{kg}$, and the maximum tolerated dose is between 2.5 and $7 \mathrm{mmol} / \mathrm{kg}$. ${ }^{39}$

\section{Tumor/leg immobilization and image registration}

To facilitate registration of the tumor location in the MRI, EPR $\mathrm{pO}_{2}$ imager, and XRAD225cx treatment system, the tumor-bearing mouse leg was immobilized in a soft rubber, half-circumferential vinyl polysiloxane dental mold cast (GC America) that did not obstruct blood flow in the animal. The fiducials for image registration were inserted into the mold before cure. ${ }^{28}$ They contained a 2-mM water solution of trityl spin probe detectable with CT, MRI, and EPR. ${ }^{40}$ The ArbuzGUI MATLAB toolbox was used for image registration (http://epri.uchicago.edu/page/softwaredownload).

\section{Selection of hypoxia-target regions}

A key advance in the present work is the capability of treating all the hypoxic voxels identified in the tumor by the $\mathrm{EPR} \mathrm{pO}_{2}$ images ( $\mathrm{pO}_{2} \leq 10$ torr), the PTV $\mathrm{HR}$. As indicated in Figure 1, regions as small as a single voxel are selected for treatment, including those voxels 
not in the central plane of the tumor. Not all hypoxic volumes are tumor associated. For animals with fur, the high metabolism of dense hair follicles in the thick hypovascular hide can be hypoxic. Only hypoxic superficial voxels within 1 voxel of the tumor volume were deemed hypoxic targets.

\section{Radiation block fabrication}

Radiation blocks conformal with a PTV $\mathrm{HR}_{\mathrm{HR}}$ or $\mathrm{PTV}_{\mathrm{LR}}$ aperture for the XRAD225Cx (Materials E2; available online at https://dx.doi.org/10.1016/j.ijrobp.2018.10.041) were 3Dprinted from GMASS Tungsten Metal ABS Filament (Turner MedTech) containing acrylonitrile butadiene styrene (ABS) plastic infused with fine tungsten particles to a density of approximately $4 \mathrm{~g} / \mathrm{mL}$. This material had radiation attenuation one third that of lead. Blocks were simultaneously fabricated using 2 M2 3D printers (MakerGear, Beachwood, Ohio) in approximately 10 minutes. Typical dose distributions from hypoxic boost and welloxygenated boost apertures are shown in quantitative Gafchromic EBT3 medical dosimetry film exposures in Fig. E10 (available online at https://dx.doi.org/10.1016/j.ijrobp.

2018.10.041).

\section{Radiation boost shape calculation}

The $\mathrm{PTV}_{\mathrm{HR}}$ accounted for errors in positioning of the hypoxic tumor and image registration for each animal tumor and its hypoxic regions by expanding the hypoxic regions by $1.2 \mathrm{~mm}$ (Fig. 1A). The hypoxia-avoiding beams were treated as a ribbon around each hypoxic volume expanded by $0.6 \mathrm{~mm}$. The outer edge of the ribbon was chosen to provide a PTV $\mathrm{LR}_{\mathrm{LR}}$ area equal to that of the $\mathrm{PTV}_{\mathrm{HR}}$ (Fig. 1B).

\section{Determining the hypoxic boost region $\left(\mathrm{PTV}_{\mathrm{HR}}\right)$ or well-oxygenated boost regions (PTV $\left.\mathrm{LR}_{\mathrm{L}}\right)$}

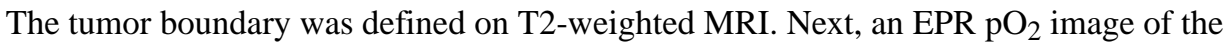
tumor-bearing leg was obtained and registered with the MRI. ${ }^{41}$ A digitally reconstructed radiograph of the $\mathrm{pO}_{2}$ image provided the shape of the conformal beam for the boost experiment shown in Figure $1 \mathrm{~A}$ with the $\mathrm{pO}_{2}$ image slice orthogonal to the radiation beam direction at tumor midplane. The red outlines are the projection of all hypoxic regions on the treatment midplane. A 1.2-mm margin shown as a black outline defines the $\mathrm{PTV}_{\mathrm{HR}}$ and the block aperture. The insert in the left upper corner highlights to boost aperture shape. A similar plan for the hypoxia avoidance $\mathrm{PTV}_{\mathrm{LR}}$ boost with a 0.6-mm hypoxia margin is shown in Figure 1B with the outer edge adjusted to equalize the area of the aperture approximately to that of the hypoxic boost.

\section{Radiation treatment}

The gantry-mounted XRAD225Cx radiator delivered $225 \mathrm{kV}$ bremsstrahlung for treatment (Precision X-Ray, North Branford, CT Fig. E2, available online at https://dx.doi.org/ 10.1016/j.ijrobp.2018.10.041 ). The XRAD225Cx was also used in 40-kVp mode as a planning cone beam $\mathrm{CT}$ for boost registration with MRI and $\mathrm{EPR} \mathrm{pO}_{2}$ images. First, the $\mathrm{TCD}_{15}(22.5 \mathrm{~Gy})$ was given to the whole tumor plus a $6 \mathrm{~mm}$ margin using opposed anterior and posterior beams. The 13-Gy boost dose to either hypoxic $\mathrm{PTV}_{\mathrm{HR}}$ or well-oxygenated $\mathrm{PTV}_{\mathrm{LR}}$ was delivered using opposed oblique beams (Fig. 1C) whose angle was chosen to 
give the most compact hypoxic area from a sample of 5 angles separated by 72 degrees, including a straight anterior angle. Dose and spatial distributions were validated using Gafchromic EBT3 medical dosimetry film (Ashland, Fiskeville, RI; http:// www.gafchromic.com/documents/EBT3_Specifications.pdf) ${ }^{41}$ calibrated with an ion chamber.

The radiation blocks for radiation boost were $3 \mathrm{D}$ printed as soon as the $\mathrm{EPR} \mathrm{pO}_{2}$ image was completed, including all tumor voxels with $\mathrm{pO}_{2} \leq 10$ torr. The blocks for the randomly chosen hypoxic boost $\left(\mathrm{PTV}_{\mathrm{HR}}\right)$ or welloxygenated boost $\left(\mathrm{PTV}_{\mathrm{LR}}\right)$ were installed into the irradiator and treated within a median of 65 minutes (Fig. E5; available online at https:// dx.doi.org/10.1016/j.ijrobp.2018.10.041). The overall duration of the experiment from the beginning of MRI until the end of radiation was approximately 4 hours.

\section{Statistical analysis}

For local tumor control in mice randomized to receive (1) a radiation boost to all hypoxic tumor $\left(\mathrm{PTV}_{\mathrm{HR}}\right)$ or $(2)$ a radiation boost to an equal volume of tumor that was well oxygenated, $\mathrm{PTV}_{\mathrm{LR}}$ was compared using Kaplan-Meier survival curves evaluated for a trial period of 90 days. Mice were censored from the study as noted in the following section. Statistical significance of the difference between the Kaplan-Meier curves was evaluated using a log-rank test from MedCalc.

\section{Results}

Seventy-eight animals were entered into the study. A total of 24 mice were then removed from the study: 14 with no detectable hypoxia at the experiment start, 3 with $\mathrm{pO}_{2} \leq 10$ torr hypoxic fraction $>0.5$ (because they could not be treated with an equal volume of welloxygenated tumor), and 7 because of failure in the process of radiation. This left 54 for randomization. Ten animals were censored from the study and immediately euthanized at the time of appearance of metastatic secondary tumors sites. Three animals were censored immediately at death before 90 days from effects not related to tumor regrowth. Data shown in the supplementary materials indicate that the well-oxygenated tumor fraction treated in the hypoxic boost cohort was approximately twice that in the well-oxygenated boost cohort (Fig. E8; available online at https://dx.doi.org/10.1016/j.ijrobp.2018.10.041). Welloxygenated tumor in the hypoxic boost cohort appeared in front of or behind the hypoxic tumor that was targeted. Hypoxia was rigorously avoided in the well-oxygenated boost cohort.

A total of 54 animals were included in the Kaplan-Meier analysis; these included all animals that survived free of tumor for 90 days, local failures, and those animals censored at the time of event not related to local failure. Figure 2 shows the Kaplan-Meier plot of the probability of animal survival, free of local tumor recurrence, plotted as a function of time in days after each treatment. It compares radiation control of FSa fibrosarcomas treated with hypoxia boost and with hypoxia avoiding boost. All animals with local failure triggered a decrement in the survival probability curve at the time of the failure. The log-rank analysis shows that the time to local tumor recurrence is significantly different in the 2 treatment groups ( $P=$. 04). 
Table 1 demonstrates the results of random assignment of tumors for 2 treatment groups. The tumors in both groups show approximately equal 10 torr hypoxic fraction (HF10), volume, and growth rate before treatment.

\section{Discussion}

Hypoxia-mediated resistance to radiation has been known for over a century. ${ }^{42}$ However, this work presents the first data demonstrating that targeting hypoxic tumor regions with radiation improves the control of mammalian tumors relative to a control boost to nonhypoxic regions of the tumor, albeit with a single tumor type. Previous data with approximately $85 \%$ tumor hypoxia coverage failed to provide a significant difference between hypoxia boost and hypoxia avoidance boost treatments (Figs. E4 and E7; available online at https://dx.doi.org/10.1016/j.ijrobp.2018.10.041). The increase in tumor control treating all hypoxia validates EPR $\mathrm{pO}_{2}$ images as a quantitative assessment of biologically relevant $\mathrm{pO}_{2}$. $\mathrm{EPR} \mathrm{pO}_{2}$ images can be used in preclinical models as a legitimate imaging modality for defining hypoxic tumor subregions as targets for dose painting targeting. It demonstrates that conformal radiation using 3D-printed blocks enhances the accuracy of the treatment of small hypoxic volumes in mouse tumors.

An immediate concern arises in data presented in the supplementary material (Fig. E1; available online at https://dx.doi.org/10.1016/j.ijrobp.2018.10.041) showing that separate single-dose control experiments established $35.5 \mathrm{~Gy}$ as sufficient for $95 \%$ tumor control, whereas the hypoxic boost provided only $60 \%$ control. A clear explanation is that factors other than the $\mathrm{EPR} \mathrm{pO}_{2}$ image-defined hypoxia contribute to apparent resistance. Transient changes in $\mathrm{pO}_{2}$ or cycling hypoxia altering $\mathrm{pO}_{2}$ during the 1-hour delay between $\mathrm{pO}_{2}$ image and boost treatment could be a source of confounding variation. Dewhirst et al. ${ }^{8}$ show substantial transient changes in a rat fibrosarcoma grown in a window chamber with 60- to 90-minute cycle time. In Fourier analysis of the frequency of $\mathrm{pO}_{2}$ changes, there is significant power in the frequencies of 2 to 3 per hour, although there may be major components that are on the order of days that are difficult to measure. ${ }^{43-45}$ Reducing delay between image acquisition and treatment should increase tumor control, if this is a major factor.

Another major factor that might explain the lower-than-expected tumor control is seen in the actual doses delivered to small hypoxic volumes. The radiation block in Figure E10A (available online at https://dx.doi.org/10.1016/j.ijrobp.2018.10.041) shows the dose delivered to a hypoxic boost with quantitative Gafchromic film. The large region of the boost has uniform dose. Small, disconnected regions of hypoxia appear underdosed, qualitatively by as much as $30 \%$. Given the prior failure to treat hypoxic voxels completely (Fig. E4; available online at https://dx.doi.org/10.1016/j.ijrobp.2018.10.041), this provides an additional explanation for the discrepancy between the expected control and the less than $95 \%$ control.

As noted in the Results, the assumed similar integral dose from the hypoxic boost compared with the well-oxygenated tumor boost is not entirely accurate, with a higher integral dose 
delivered with the hypoxic boost. This might bias the results toward improved hypoxic boost tumor control.

The partial-volume effects from EPR imaging result from the use of Nyquist frequency cutoff of 0.5 in the back-projection apodization to reduce high-frequency noise. The basic image voxel size from binning is $0.67 \mathrm{~mm}$ so that the smearing of the image information gives a true resolution of $1.3 \mathrm{~mm}$. This is the minimum linear distance to which the EPR imaging is sensitive.

There are a number of technical aspects of our experiments that can be improved. Considering the small volumes of the tumors (mean, $\sim 400 \mu \mathrm{L}$ ), the radiation delivery required submillimeter accuracy. This is defined by the accuracy of $\mathrm{pO}_{2}$ images and image registration precision. Some hypoxia pockets are likely to be too small to be identified by the available EPR imaging technology (microhypoxia). ${ }^{46,47}$

EPR $\mathrm{pO}_{2}$ images can enhance hypoxia ${ }^{18} \mathrm{~F} 2^{\prime}$-nitroimidazole based $\mathrm{PET}^{5}$ for hypoxiatargeted dose painting. ${ }^{21}$ The comparison of preclinical EPR $\mathrm{pO}_{2}$ images with ${ }^{18}$ Fnitroimidazole PET images corrected by registered dynamic contrast-enhanced MRI and chemical exchange saturation transfer $\mathrm{pH}$ measuring MRI can form the basis by which such PET thresholds can be modified for more accurate local hypoxia targeting.

If further work confirms that quantitative $\mathrm{EPR} \mathrm{pO}_{2}$ images consistently define resistant tumor regions, then it might be possible to implement true IMRT dose painting to reduce radiation dose delivered to well-oxygenated tumor volumes. It will confirm that a major source of cancer radiation treatment failure is localizable resistant hypoxic tumor regions. The difference in survival in our experiment, $60 \%$ versus $28 \%$, is roughly the difference in tumor control found in patient data between those with significant hypoxia versus those with little hypoxia (mean $\mathrm{pO}_{2}$ greater or less than 10 torr). ${ }^{10-12}$ These data also suggest that identifying hypoxic regions of tumors and treating them with extra dose might benefit patients, with minimal increase in complications. However, much more work is necessary to confirm these implications.

A more basic biologic question that this work begins to answer is the importance of small subvolumes within a tumor in determining tumor curability. Dewhirst et $\mathrm{al}^{22}$ have questioned "whether the link between hypoxia and radioresistance is completely explainable by the oxygen enhancement effect or, rather, whether hypoxia also influences radiosensitivity through biological effects." Gillies et al ${ }^{48,49}$ have characterized tumor subvolumes as habitats, regions of clonal homogeneity likely consisting of cells that are the progeny of a resistant clone, distinct from other regions of a clonally heterogeneous tumor.

\section{Conclusion}

This work is limited to a single murine tumor type, albeit one that has been used extensively in understanding tumor hypoxia. Issues of transient hypoxia and tiny apertures to treat all hypoxic tumor subvolumes that might underdose tumor hypoxia could explain why tumor control is only $60 \%$ when the boost dose is sufficient to provide a $95 \%$ control when given to the whole tumor. Other sources of tumor resistance might also explain this. 
This work suggests that hypoxia develops in specific subvolumes within a tumor and that specifically targeting these subvolumes can lead to more effective IMRT radiation therapy, which will reduce dose to nearby critical, quality-of-life-related structures. It suggests that selection of hypoxic tumor regions that can be exposed safely to higher radiation dose might improve tumor control and enhance the therapeutic ratio.

\section{Supplementary Material}

Refer to Web version on PubMed Central for supplementary material.

\section{Acknowledgments-}

Funding was provided by the National Institutes of Health grants P41 EB002034, R01 CA098575 and R50

CA211408. We thank John L. Humm for many useful suggestions. Mihai Giurcanu provided statistical assistance.

\section{References}

1. De Vita VT, Lawrence TS, Rosenberg SA. De Vita, Hellman and Rosenberg's Cancer: Principles and Practice of Oncology. 10th ed. Philadelphia: Lippincott Williams and Wilkins; 2014.

2. Leibel SA, Fuks Z, Zelefsky MJ, et al. Technological advances in external-beam radiation therapy for the treatment of localized prostate cancer. Semin Oncol 2003;30:596-615. [PubMed: 14571409]

3. Mundt A, Roeske J. Intensity Modulated Radiation Therapy. Hamilton, Ontario: B.C. Decker; 2005.

4. Kataria T, Sharma K, Subramani V, et al. Homogeneity index: An objective tool for assessment of conformal radiation treatments. J Med Phys 2012;37:207-213. [PubMed: 23293452]

5. Ling CC, Humm J, Larson S, et al. Towards multidimensional radiotherapy (MD-CRT): Biological imaging and biological conformality. Int J Radiat Oncol Biol Phys 2000;47:551-560. [PubMed: 10837935]

6. Overgaard J Hypoxic radiosensitization: Adored and ignored. J Clin Oncol 2007;25:4066-4074. [PubMed: 17827455]

7. Vaupel P, Hockel M, Mayer A. Detection and characterization of tumor hypoxia using pO2 histography. Antioxid Redox Signal 2007;9: 1221-1235. [PubMed: 17536958]

8. Dewhirst MW, Cao Y, Moeller B. Cycling hypoxia and free radicals regulate angiogenesis and radiotherapy response. Nature Reviews Cancer 2008;8:425-437. [PubMed: 18500244]

9. Warburg O The Metabolism of Tumours. London: Constable \& Co., Ltd.; 1930.

10. Nordsmark M, Overgaard M, Overgaard J. Pretreatment oxygenation predicts radiation response in advanced squamous cell carcinoma of the head and neck. Radiother Oncol 1996;41:31-39. [PubMed: 8961365]

11. Brizel DM, Scully SP, Harrelson JM, et al. Tumor oxygenation predicts for the likelihood of distant metastases in human soft tissue sarcoma. Cancer Res 1996;56:941-943. [PubMed: 8640781]

12. Hockel M, Schlenger K, Aral B, et al. Association between tumor hypoxia and malignant progression in advanced cancer of the uterine cervix. Cancer Res 1996;56:4509-4515. [PubMed: 8813149]

13. Coleman CN. Chemical sensitizers and protectors. Int J Radiat Oncol Biol Phys 1998;42:781-783. [PubMed: 9845095]

14. Overgaard J Hypoxic modification of radiotherapy in squamous cell carcinoma of the head and neck-a systematic review and meta-analysis. Radiother Oncol 2011;100:22-32. [PubMed: 21511351]

15. Peeters SG, Zegers CM, Lieuwes NG, et al. A comparative study of the hypoxia PET tracers [(1) (8)F]HX4, [(1)(8)F]FAZA, and [(1)(8)F] FMISO in a preclinical tumor model. Int J Radiat Oncol Biol Phys 2015;91:351-359. [PubMed: 25491505] 
16. Servagi-Vernat S, Differding S, Hanin FX, et al. A prospective clinical study of (1)(8)F-FAZA PET-CT hypoxia imaging in head and neck squamous cell carcinoma before and during radiation therapy. Eur J Nucl Med Mol Imaging 2014;41:1544-1552. [PubMed: 24570097]

17. Chitneni SK, Bida GT, Yuan H, et al. 18F-EF5 PET imaging as an early response biomarker for the hypoxia-activated prodrug SN30000 combined with radiation treatment in a non-small cell lung cancer xenograft model. J Nucl Med 2013;54:1339-1346. [PubMed: 23740105]

18. Rischin D, Hicks RJ, Fisher R, et al. Prognostic significance of [18F]-misonidazole positron emission tomography-detected tumor hypoxia in patients with advanced head and neck cancer randomly assigned to chemoradiation with or without tirapazamine: A substudy of Trans-Tasman Radiation Oncology Group Study 98.02. J Clin Oncol 2006; 24:2098-2104. [PubMed: 16648512]

19. Silvoniemi A, Suilamo S, Laitinen T, et al. Repeatability of tumour hypoxia imaging using [(18)F]EF5 PET/CT in head and neck cancer. Eur J Nucl Med Mol Imaging 2018;45:161-169. [PubMed: 29075831]

20. Vera P, Thureau S, Chaumet-Riffaud P, et al. Phase II study of a radiotherapy total dose increase in hypoxic lesions identified by $18 \mathrm{~F}-$ misonidazole PET/CT in patients with non-small cell lung carcinoma (RTEP5 Study). J Nucl Med 2017;58:1045-1053. [PubMed: 28254869]

21. Grkovski M, Schwartz J, Rimner A, et al. Reproducibility of (18)F-fluoromisonidazole intratumour distribution in non-small cell lung cancer. EJNMMI Res 2016;6:79. [PubMed: 27822900]

22. Moeller BJ, Richardson RA, Dewhirst MW. Hypoxia and radiotherapy: Opportunities for improved outcomes in cancer treatment. Cancer Metastasis Rev 2007;26:241-248. [PubMed: 17440683]

23. Carlin S, Humm JL. PET of hypoxia: Current and future perspectives. J Nucl Med 2012;53:11711174. [PubMed: 22789676]

24. Halpern HJ, Yu C, Peric M, et al. Oxymetry deep in tissues with low-frequency electron paramagnetic resonance. Proc Natl Acad Sci U S A 1994;91:13047-13051. [PubMed: 7809170]

25. Kuppusamy P, Shankar RA, Zweier JL. In vivo measurement of arterial and venous oxygenation in the rat using 3D spectral-spatial electron paramagnetic resonance imaging. Phys Med Biol 1998;43: 1837-1844. [PubMed: 9703045]

26. Matsumoto K-I, Subramanian S, Murugesan R, et al. Spatially resolved biologic information from in vivo EPRI, OMRI, and MRI. Antioxid Redox Signal 2007;9:1125-1142. [PubMed: 17571957]

27. Epel B, Bowman MK, Mailer C, et al. Absolute oxygen R1 imaging in vivo with pulse electron paramagnetic resonance. Magn Reson Med 2014;72:362-368. [PubMed: 24006331]

28. Elas M, Bell R, Hleihel D, et al. Electron paramagnetic resonance oxygen image hypoxic fraction plus radiation dose strongly correlates with tumor cure in FSa fibrosarcomas. Int J Radiat Oncol Biol Phys 2008;71:542-549. [PubMed: 18474313]

29. Elas M, Hleihel D, Barth ED, et al. Where it's at really matters: in situ in vivo vascular endothelial growth factor spatially correlates with electron paramagnetic resonance $\mathrm{pO} 2$ images in tumors of living mice. Mol Imaging Biol 2011;13:1107-1113. [PubMed: 20960236]

30. Elas M, Magwood JM, Butler B, et al. EPR Oxygen Images Predict Tumor Control by a 50\% Tumor Control Radiation Dose. Cancer Res 2013;73:5328-5335. [PubMed: 23861469]

31. Matsumoto S, Batra S, Saito K, et al. Antiangiogenic agent sunitinib transiently increases tumor oxygenation and suppresses cycling hypoxia. Cancer Res 2011;71:6350-6359. [PubMed: 21878530]

32. Lin Z, Mechalakos J, Nehmeh S, et al. The influence of changes in tumor hypoxia on dose-painting treatment plans based on 18F-FMISO positron emission tomography. Int J Radiat Oncol Biol Phys 2008;70: 1219-1228. [PubMed: 18313529]

33. Haney CR, Parasca AD, Ichikawa K, et al. Reduction of image artifacts in mice by bladder flushing with a novel double-lumen urethral catheter. Mol Imaging 2006;5:175-179. [PubMed: 16954032]

34. Suit HD, Suchato C. Hyperbaric oxygen and radiotherapy of a fibrosarcoma and of a squamouscell carcinoma of C3H mice. Radiology 1967;89:713-719. [PubMed: 6059611]

35. Mustafi D, Zamora M, Fan X, et al. MRI accurately identifies early murine mammary cancers and reliably differentiates between in situ and invasive cancer: Correlation of MRI with histology. NMR Biomed 2015;28:1078-1086. [PubMed: 26152557] 
36. Epel B, Sundramoorthy SV, Mailer C, et al. A versatile high speed 250-MHz pulse imager for biomedical applications. Concepts Magn Reson Part B Magn Reson Eng 2008;33B:163-176. [PubMed: 19924261]

37. Froncisz W, Hyde JS. The loop-gap resonator: A new microwave lumped circuit ESR sample structure. J Magnetic Resonance 1982;47: 515-521.

38. Kuzhelev AA, Trukhin DV, Krumkacheva OA, et al. Room-temperature electron spin relaxation of triarylmethyl radicals at the X- and Q-bands. J Phys Chem B 2015;119:13630-13640. [PubMed: 26001103]

39. Krishna MC, Subramanian S, Kuppusamy P, et al. Magnetic resonance imaging for in vivo assessment of tissue oxygen concentration. Semin Radiat Oncol 2001;11:58-69. [PubMed: $11146043]$

40. Reddy TJ, Iwama T, Halpern HJ, et al. General synthesis of persistent trityl radicals for EPR imaging of biological systems. J Org Chem 2002;67:4635-4639. [PubMed: 12098269]

41. Epel B, Maggio M, Pelizzari C, et al. Electron Paramagnetic Resonance pO2 Image Tumor Oxygen-Guided Radiation Therapy Optimization. Adv Exp Med Biol 2017;977:287-296. [PubMed: 28685458]

42. Schwarz G Uber Desensibilisierung gegen Rontgen- und Radiumstrahlen. Munchner Medizinische Wochenschrift 1909;56:1217-1218.

43. Braun RD, Lanzen JL, Dewhirst MW. Fourier analysis of fluctuations of oxygen tension and blood flow in R3230Ac tumors and muscle in rats. Am J Physiol 1999;277(2 Pt 2):H551-568. [PubMed: 10444480]

44. Dewhirst MW. Relationships between cycling hypoxia, HIF-1, angiogenesis and oxidative stress. Radiat Res 2009;172:653-665. [PubMed: 19929412]

45. Cardenas-Navia LI. Tumor-dependent kinetics of partial pressure of oxygen fluctuations during air and oxygen breathing. Cancer Res 2004;64:6010-6017. [PubMed: 15342381]

46. Raleigh JA, Chou SC, Bono EL, et al. Semiquantitative immunohistochemical analysis for hypoxia in human tumors. Int J Radiat Oncol Biol Phys 2001;49:569-574. [PubMed: 11173156]

47. Cline JM, Rosner GL, Raleigh JA, et al. Quantification of CCI-103F labeling heterogeneity in canine solid tumors. Int J Radiat Oncol Biol Phys 1997;37:655-662. [PubMed: 9112464]

48. Chaudhury B, Zhou M, Goldgof DB, et al. Heterogeneity in intratumoral regions with rapid gadolinium washout correlates with estrogen receptor status and nodal metastasis. J Magn Reson Imaging 2015;42:1421-1430. [PubMed: 25884277]

49. Gatenby RA, Gillies RJ. A microenvironmental model of carcinogenesis. Nat Rev Cancer 2008;8:56-61. [PubMed: 18059462] 


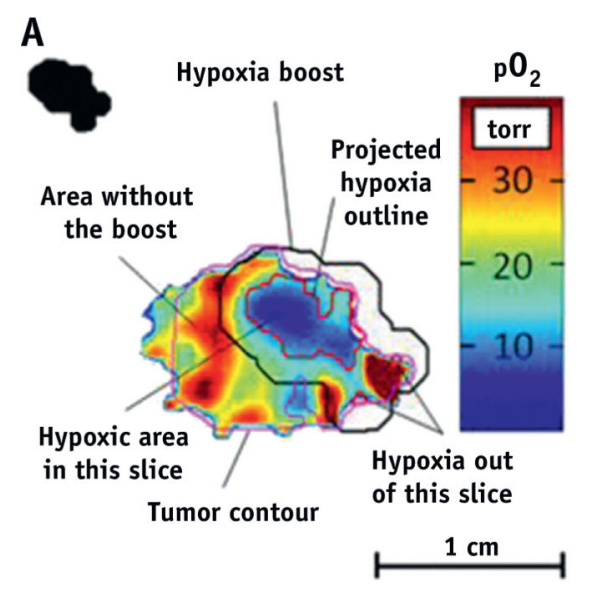

B

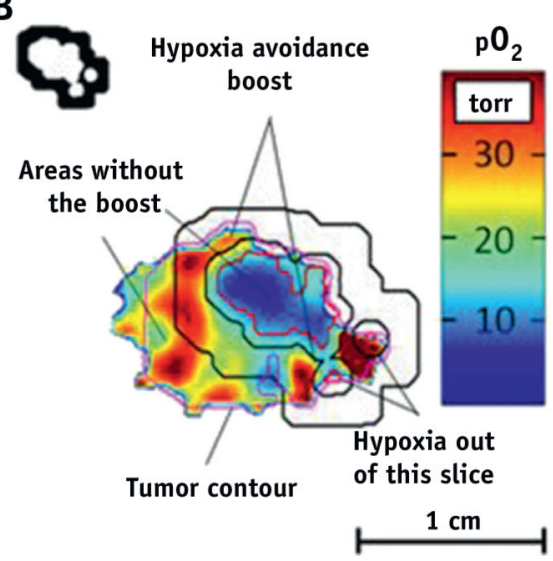

C

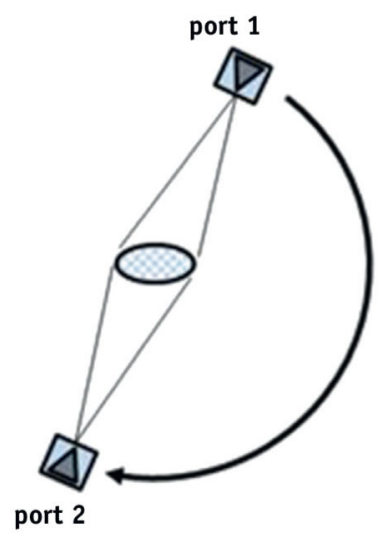

Fig. 1.

Radiation treatment plans and delivery scheme. (A) EPR $\mathrm{pO}_{2}$ image slice orthogonal to the radiation beam showing hypoxia boost treatment plan. (B) The same EPR $\mathrm{pO}_{2}$ image slice showing hypoxia avoidance boost. Magenta contour: MRI-defined tumor margin. Red contour: projection of all in-tumor hypoxic volumes onto the EPR image plane. Black contours - radiation treatment beam shape including additional setup uncertainty margins. The area of the hypoxia avoiding boost equals the area of the hypoxia boost. Note in both (A) and (B). the islands of hypoxia out of the plane derived from the DRR of the whole tumor volume as well as the margin about the hypoxia, $1.2 \mathrm{~mm}$ for the hypoxia boost and $0.6 \mathrm{~mm}$ for the hypoxia avoiding boost. Upper Left corners of (A) and (B): Black shapes of hypoxic boost (A) and well oxygenated boost (B) apertures. (C) Illustration of opposed field radiation boost treatment with XRAD225Cx gantry-mounted X-ray machine. Each field was treated with half of the total boost dose. 


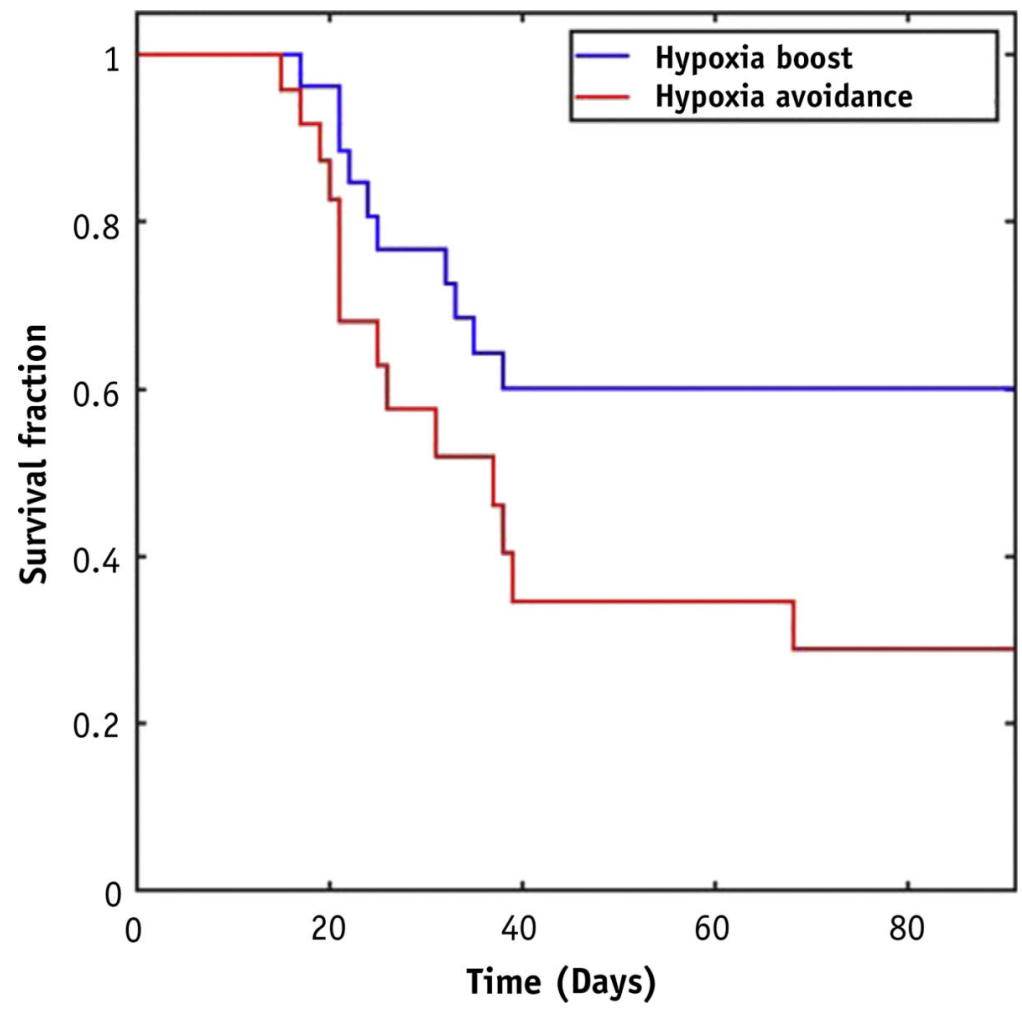

Fig. 2.

Kaplan-Meier survival plot comparing conformal hypoxia boost with hypoxia avoidance boost. The two treatments differ significantly $(P=.04)$ demonstrating the therapeutic efficacy of hypoxia guided radiation. 
